\title{
How the first generations of luminous baryons established the X-ray and UV backgrounds
}

\author{
Andrei Mesinger*i \\ Princeton University \\ E-mail: mesinger@astro.princeton.edu
}

The first generations of astrophysical objects made a substantial impact on our Universe with their radiation. X-rays from the first sources, with large mean free paths, likely quickly heated the intergalactic medium (IGM). The second generation of $21 \mathrm{~cm}$ instruments can provide a unique view into this early epoch. The early stages of reionization likely followed, driven by so-called "minihalos", i.e. molecularly-cooled halos. These small halos were susceptible to complex feedback mechanisms, especially from the soft-UV background which preceded reionization, resulting in complex and possibly extended early stages of reionization. When atomically-cooled galaxies emerged as the dominant ionizers, reionization could proceed more rapidly, with these being less sensitive to radiative feedback than previously thought. Reionization could have slowed in the final stages when the ionized bubbles grew larger than the separation of Lyman limit absorption systems (LLSs). The final stages likely involved the photo-evaporation of LLSs, which by then regulated the rise of the UV background. I discuss the theoretical underpinnings of this narrative, as well as how future $21 \mathrm{~cm}$ observations may help shed light on the outstanding uncertainties.

Cosmic Radiation Fields: Sources in the early Universe

November 9-12, 2010

Desy, Germany

\footnotetext{
* Speaker.

${ }^{\dagger}$ Hubble Fellow
} 


\section{Intro}

The dawn of the first astrophysical objects and the eventual reionization of the Universe is of fundamental importance, offering insight into many astrophysical processes. Also, since observational surveys only pick out the rarest, most luminous objects, cosmic radiation fields and their effects on the intergalactic medium (IGM) may our only way of studying the majority of our galactic ancestors. Recent theoretical and observational advances are starting to shed light on a complex and extended reionization epoch. Here I sketch-out three likely stages of this process. I briefly discuss the potential of the redshifted $21 \mathrm{~cm}$ signal to probe not only reionization, but also the pre-reionization epochs. However, interpreting the upcoming observations requires efficient modeling tools, such as the recently developed $21 \mathrm{cmFAST}$.

\section{Once upon a time... the story of cosmic dawn and reionization}

\subsection{Early stages}

Hierarchal structure formation implies that the first astrophysical objects are likely hosted by so-called minihalos (with virial temperatures $T_{\text {vir }} \lesssim 10^{4} \mathrm{~K}$ ). Cooling via atomic hydrogen is inefficient at such low temperatures, so baryons condense into these halos via the molecular hydrogen $\left(\mathrm{H}_{2}\right)$ cooling channel. Without metals to aid in cooling and fragmentation, the first generations of stars (so-called PopIII stars) are likely massive (with masses of $\sim 100 M_{\odot} ;[1,5,81]$ ), yet shortlived (with lifetimes of a few Myr; [61, 62]).

PopIII stars are likely to have very different properties from "normal" PopII stars, with harder spectra, a factor of ten times more ionizing photons per baryon [61], and various exotic, massdependent fates (e.g., [29]). Accurately determining their properties, formation efficiency and initial mass functions (IMFs) requires detailed numerical simulations and costly explorations of parameter space (e.g., [73]).

These prehistoric giants have complex interactions with their surroundings, since star formation in such small-mass halos is susceptible to feedback mechanisms. Modeling these requires introducing parameterized prescriptions for stellar properties and extending simulations to larger, $\sim 1 \mathrm{Mpc}$ scales. Feedback processes can come in three forms: (1) mechanical: supernovae ( $\mathrm{SNe}$ ) can blow out gas from the surrounding shallow potential well, delaying local star formation until gas is re-accreted (e.g., $[74,75])$; (2) chemical: evolution in the IMF depends on metal enrichment $[68,65,63]$, which is very inhomogeneous making it necessary to model the enrichment on large scales (e.g., [70]); and (3) radiative: radiation in various bands can either promote (positive feedback) or suppress (negative feedback) future star formation.

Because it acts on very large scales and involves a large dynamic range in energy, it is likely that radiative feedback is the most pivotal and complex of the feedback mechanisms. Positive radiative feedback can result when the enhanced free-electron fraction from ionizing photons (e.g., [56]) or hydrodynamical shocks [64] catalyzes the formation of $\mathrm{H}_{2}$, thereby enhancing the $\mathrm{H}_{2}$ cooling channel. Negative feedback can result from heating by ionizing radiation which can photoevaporate gas in low-mass halos (e.g., [11]). Also, an active background of Lyman-Werner (LW) radiation (with photon energies in the $11.18-13.6 \mathrm{eV}$ range) can dissociate $\mathrm{H}_{2}$, thus decreasing the gas's cooling capabilities (e.g., [28]). 
The first astrophysical objects can emit radiation in various bands. X-rays, generated from accretion onto remnant black holes or massive X-ray binaries, have long mean free paths, but their feedback effects seem to be mild [38, 34]. However, they can be responsible for early IGM heating and some degree of reionization (e.g., $[9,59,50])$. Feedback resulting from a LW background is by definition negative, and can delay star formation in low mass halos, depending on the strength of the background [37, 38, 82, 58]. Also depending on its strength, a transient (due to the short lifetimes of PopIII stars) ionizing UV background (UVB) can have various feedback regimes [57, $46,47,75,74]$, especially in conjunction with the negative feedback effects of a LW background $[46,47,75]$. Despite this complexity, it is likely that the final suppression of star formation inside minihalos was regulated by the build-up of persistent UV or LW backgrounds before the bulk of reionization $[25,26]$. However, the complexity of radiative feedback and/or an early X-ray background could precipitate an extended early reionization epoch, which could allow a significant amount of reionization to occur at late times $(z \sim 6)$, and still be consistent with constraints from the Wilkinson Microwave Anisotropy Probe (WMAP) [35].

\subsection{Middle stages}

After the initial, "fireworks" stage of PopIII-driven reionization, longer-lived stars begin to establish persistent UVBs. Reionization proceeds in an "inside-out" fashion on large-scales, with biased sources driving HII regions to tens of Mpc in size (e.g., [21, 19, 84]). Larger, atomicallycooled halos $\left(T_{\mathrm{vir}} \gtrsim 10^{4} \mathrm{~K}\right)$ start dominating the ionization budget. These are likely too faint to image directly with current or upcoming instruments (e.g., [60, 80]), although we can get close with gravitational lensing surveys (e.g., $[67,52,79]$ ). Likewise, because of the inside-out nature of reionization, most astrophysical objects during the epoch of reionization find themselves inside large HII regions, largely unaffected by the neutral IGM even when it still occupies a substantial volume filling fraction. Thus most of the current observational probes of reionization (derived from quasar proximity regions, damping wings in quasar and gamma ray burst (GRB) spectra, Ly $\alpha$ emitter (LAE) number density and clustering properties; see below) are most effective back when the Universe is predominantly neutral.

Aside from these observational challenges, there are substantial theoretical hurdles to overcome when studying the middle stages of reionization. Ionization structures during this epoch can span volumes over four magnitudes larger than the first-stars simulations discussed in the previous section (see Fig. 1). To statistically capture the middle stages of reionization, simulation boxes need to be hundreds of Mpc in length, yet still be able to account for ionizing radiation from smallmass halos. The required dynamic range is daunting, and state-of-the-art simulations are forced to ignore minihalos and photon sinks, while also using approximate prescriptions to assign ionizing luminosities to source halos (see the recent review in [72]). More approximate techniques have recently been developed in order to overcome the challenge of such a large range of scales, including sub-grid [33, 43] and semi-numerical [83, 49, 23, 2, 7, 69, 50] models.

Additionally, our poor understanding of the early Universe means that large explorations of parameter space are required for any robust conclusion. Analytic models have been very useful in this respect, predicting that HII morphology is not very sensitive to redshift, when normalized to the same mean neutral fraction, $\bar{x}_{\mathrm{HI}}$, and also that sources are more important that ionizing sinks in this regime $[21,19]$. These predictions were confirmed by the first numerical parameter studies 


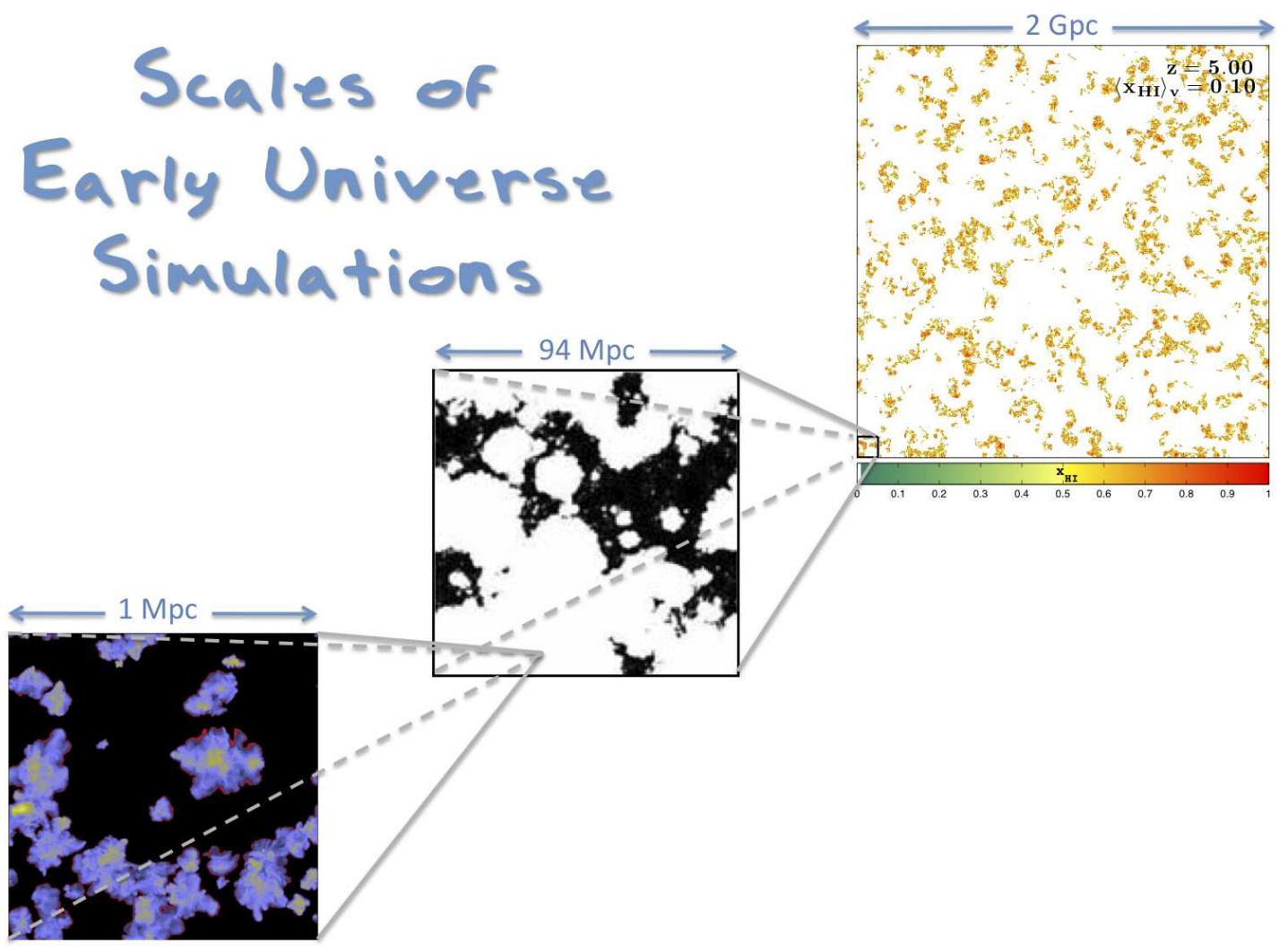

Figure 1: Scales of some early Universe simulations, with box sizes indicated in comoving units. Slices correspond to metallicity maps from [76], and ionization structure from [43] and [45] (left to right). The left and middle panels roughly correspond to the current maximum box sizes of state-of-the-art numerical simulations of the first stars and the middle stages of reionization, respectively. This figure highlights the daunting dynamic range of cosmological processes such as reionization. In principle, one should continue "zooming-in" down to stellar scales (e.g., [61, 29]).

in [43]. Another parameter study, [48], combined 1D hydrodynamical collapse simulations with large-scale 3D semi-numerical simulations of the UVB during reionization, and concluded that radiative feedback does not play a major role during this epoch. Results of such parameter studies allow us to simplify various theoretical problems.

\subsection{Final stages}

As reionization progresses, HII regions grow to be larger than the mean free path of ionizing photons through them, requiring even larger simulation boxes (see Fig. 1). In this final regime, sinks of ionizing photons, such as Lyman limit systems (LLSs) could regulate the progress of reionization [18], and its morphological structure [19, 7]. These absorption systems are likely found in the filaments of the recently-ionized IGM, where the UVB is the weakest [8]. The final "overlap" stages of reionization could be delayed until either absorbers get photo-evaporated (e.g. [31]), or the remaining neutral islands get ionized from the inside. The time-scales for these processes are of order $\Delta z \sim 1$. This, combined with the large cosmic variance during this epoch (e.g., [45]), suggests 
that the overlap stage is much more gradual than was initially predicted by small-box simulations (e.g., [24]). It is also unlikely that these final stages leave a strong observational imprint, such as a sharp rise in the UVB [18]. Despite popular belief, current observations cannot rule-out a very late overlap, even at $z \sim 5[36,45,41]$.

\subsection{Observational constraints}

Several model-dependent constraints on $\bar{x}_{\mathrm{HI}}$ at $z \sim 6$ have been derived from various astrophysical probes such as: (1) the size of the proximity zone around quasars $([78,6]$, but see $[55,4,40])$; (2) a claimed detection of damping wing absorption from neutral IGM in quasar spectra $([53,54]$, but see [51]); (3) the non-detection of intergalactic damping wing absorption in a GRB spectrum ([71], but see [44]); and (4) the number density and clustering of Ly $\alpha$ emitters ([39, 27, 22, 32, 42], but see $[10,52,30])$. Additionally, a direct lower limit on $\bar{x}_{\mathrm{HI}}$ was recently derived from the dark covering fraction in the $\operatorname{Ly} \alpha$ and $\operatorname{Ly} \beta$ forests [41].

However, constraining the ionization state of the high-redshift Universe is very difficult with current observations, since they are not direct probes of HII morphology. For example, the Lyman forests begin to saturate at $z \gtrsim 5$, becoming difficult to interpret (e.g., [66, 12, 3, 36, 45]). Similarly, the dominant population of ionizing galaxies is $\sim$ two orders of magnitude fainter than current detection limits; and in any case, galaxies offer a very biased tracer of the cosmic radiation fields. Luckily, there is a plethora of upcoming observations which should help advance our understanding: $21 \mathrm{~cm}$ tomography, high-redshift IR spectra, wide-field LAE surveys, and improved E-mode CMB polarization power spectra. Of these, arguably the $21 \mathrm{~cm}$ line from neutral hydrogen provides the greatest potential for directly studying reionization in the near term.

\section{The $21 \mathrm{~cm}$ frontier and $21 \mathrm{cmFAST}$}

The cosmological $21 \mathrm{~cm}$ signal uses the $\mathrm{CMB}$ as a back-light. The offset of the $21 \mathrm{~cm}$ brightness temperature from the CMB temperature, $T_{\gamma}$, along a line of sight (LOS) at observed frequency $v$, can be written as (c.f. [20]):

$$
\delta T_{b}(v)=27 x_{\mathrm{HI}}\left(1-\frac{T_{\gamma}}{T_{\mathrm{S}}}\right)\left(1+\delta_{\mathrm{nl}}\right)\left(\frac{H}{d v_{r} / d r+H}\right)\left(\frac{1+z}{10} \frac{0.15}{\Omega_{\mathrm{M}} h^{2}}\right)^{1 / 2}\left(\frac{\Omega_{b} h^{2}}{0.023}\right) \mathrm{mK},
$$

where $x_{\mathrm{HI}}$ is the hydrogen neutral fraction, $T_{S}$ is the gas spin temperature, $\tau_{v_{0}}$ is the optical depth at the $21 \mathrm{~cm}$ frequency $v_{0}, \delta_{\mathrm{nl}}(\mathbf{x}, z) \equiv \rho / \bar{\rho}-1$ is the evolved (Eulerian) density contrast, $H(z)$ is the Hubble parameter, $d v_{r} / d r$ is the comoving gradient of the line of sight component of the comoving velocity, and all quantities are evaluated at redshift $z=v_{0} / v-1$. The above equation also assumes that $d v_{r} / d r \ll H$, which is generally true for the pertinent redshifts and scales.

The redshifted $21 \mathrm{~cm}$ line carries with it much information, containing both astrophysical $\left(x_{\mathrm{HI}}\right.$ and $T_{\mathrm{S}}$ ) and cosmological (last four terms in eq. 3.1) terms (see [16, 17]). However, in order to make sense of the upcoming observations, one must disentangle the various components. Considering the scales and uncertainties involved, this is a daunting task.

Recently "semi-numerical" tools have proven invaluable in overcoming these obstacles. These simulations use more approximate physics than numerical simulations, but are much faster. In particular, the recent launch of $21 \mathrm{cmFAST}$ [50] (http://www.astro.princeton.edu/ mesinger/Sim) 
provided the astronomical community a publicly-available tool specialized for fast, large-scale simulations of the $3 \mathrm{D}$ cosmological $21 \mathrm{~cm}$ signal. For example, a $256^{3}$ realization of reionization with $21 \mathrm{cmFAST}$ is obtained in just a few minutes on a single CPU, with every step agreeing with cosmological hydrodynamic simulations into the quasi-non-linear regime $[49,84,50]$. Such speeds facilitate rapid explorations of the vast parameter space, necessary for interpreting upcoming observations.

Most studies thus far have focused on the reionization term, $x_{\mathrm{HI}}$, making various simplifying assumptions in eq. 3.1 . The $21 \mathrm{~cm}$ signal during reionization can indeed tell us much about the relevant physical processes and intergalactic radiation fields. However, the $21 \mathrm{~cm}$ signal also offers us a precious glimpse into even earlier epochs, via the spin temperature, $T_{\mathrm{S}}$, term.

The spin temperature can be written as (e.g. [20]):

$$
T_{\mathrm{S}}^{-1}=\frac{T_{\gamma}^{-1}+x_{\alpha} T_{\alpha}^{-1}+x_{c} T_{\mathrm{K}}^{-1}}{1+x_{\alpha}+x_{c}}
$$

where $T_{\mathrm{K}}$ is the kinetic temperature of the gas, and $T_{\alpha}$ is the color temperature, which is closely coupled to the kinetic gas temperature, $T_{\alpha} \approx T_{\mathrm{K}}$ [14]. From this equation, one can see that the spin temperature interpolates between the $\mathrm{CMB}$ and the gas temperature. It does this through two coupling coefficients: (1) the collisional coupling coefficient, $x_{c}$, which requires high densities and is effective in the IGM at $z \gtrsim 40$; and (2) the Wouthuysen-Field ([77, 13]; WF) coupling coefficient which uses the Ly $\alpha$ radiation background and is effective soon after the first sources ignite. If either coefficients is high, the spin temperature approaches the kinetic temperature of the gas. Otherwise, the spin temperature approaches the CMB temperature and there is no signal.

The CMB temperature decreases as $\propto(1+z)$. After decoupling from the $\mathrm{CMB}$, the gas kinetic temperature decreases as $\propto(1+z)^{2}$, before being heated, most likely with X-rays from the first sources (see [15] and the discussion therein). Likely before the bulk of reionization, the gas is heated to temperatures far beyond the $\mathrm{CMB}, T_{\mathrm{K}} \gg T_{\gamma}$, and the spin temperature term in eq. 3.1 no longer contributes to the signal. When and where $T_{\mathrm{S}}<T_{\gamma}$, the $21 \mathrm{~cm}$ signal is seen in absorption, and when/where $T_{\mathrm{S}}>T_{\gamma}$, the signal is seen in emission.

Therefore, the $21 \mathrm{~cm}$ signal and its inhomogeneity could be a powerful probe, not only of reionization, but also of the Ly $\alpha$ background, IGM heating, and cosmology during the collisionally coupled regime before the first sources ignite. Another probe of cosmology would be available at lower redshifts (making it easier to detect) if heating completes before reionization. A movie of $21 \mathrm{~cm}$ evolution from $z=250$, computed with $21 \mathrm{cmFAST}$, is available at http://www.astro.princeton.edu/ $\sim$ mesinger/21cm_Movie.html, together with an explanation of the various interesting epochs.

\section{Conclusions}

- Milestones such as reionization are likely the only practical way of studying the primordial zoo of astrophysical objects in the near future.

- Reionization is likely extended, going through various stages.

- The cosmological $21 \mathrm{~cm}$ signal is very rich in information, containing both cosmological and astrophysical components.

- The range of scales and unknown parameter space is enormous. 
- We need efficient modeling tools, such as $21 \mathrm{cmFAST}$, to make sense of the upcoming observations.

- We are living in exciting times!

\section{Acknowledgments}

I would like to thank all of my collaborators who contributed to the science discussed here. Support for this work was provided by NASA through Hubble Fellowship grant HST-HF-51245.01-A, awarded by the Space Telescope Science Institute, which is operated by the Association of Universities for Research in Astronomy, Inc., for NASA, under contract NAS 5-26555.

\section{References}

[1] T. Abel, G. L. Bryan, and M. L. Norman. The Formation of the First Star in the Universe. Science, 295:93-98, Jan. 2002.

[2] M. A. Alvarez, M. Busha, T. Abel, and R. H. Wechsler. Connecting Reionization to the Local Universe. ApJ, 703:L167-L171, Oct. 2009.

[3] G. D. Becker, M. Rauch, and W. L. W. Sargent. The Evolution of Optical Depth in the Ly $\alpha$ Forest: Evidence Against Reionization at ž6. ApJ, 662:72-93, June 2007.

[4] J. S. Bolton and M. G. Haehnelt. The nature and evolution of the highly ionized near-zones in the absorption spectra of $\mathrm{z}^{\sim}=6$ quasars. MNRAS, 374:493-514, Jan. 2007.

[5] V. Bromm, P. S. Coppi, and R. B. Larson. The Formation of the First Stars. I. The Primordial Star-forming Cloud. ApJ, 564:23-51, Jan. 2002.

[6] C. L. Carilli et al. Ionization Near Zones Associated with Quasars at z 6. ApJ, 714:834-839, May 2010.

[7] T. R. Choudhury, M. G. Haehnelt, and J. Regan. Inside-out or outside-in: the topology of reionization in the photon-starved regime suggested by Ly $\alpha$ forest data. MNRAS, 394:960-977, Apr. 2009.

[8] D. Crociani, A. Mesinger, L. Moscardini, and S. Furlanetto. The distribution of Lyman-limit absorption systems during and after reionization. MNRAS, pages 1655-+, Oct. 2010.

[9] M. Dijkstra, Z. Haiman, and A. Loeb. A Limit from the X-Ray Background on the Contribution of Quasars to Reionization. ApJ, 613:646-654, Oct. 2004.

[10] M. Dijkstra, J. S. B. Wyithe, and Z. Haiman. Luminosity functions of Ly $\alpha$ emitting galaxies and cosmic reionization of hydrogen. MNRAS, 379:253-259, July 2007.

[11] G. Efstathiou. Suppressing the formation of dwarf galaxies via photoionization. MNRAS, 256:43P-47P, May 1992.

[12] X. Fan et al. Constraining the Evolution of the Ionizing Background and the Epoch of Reionization with z 6 Quasars. II. A Sample of 19 Quasars. AJ, 132:117-136, July 2006.

[13] G. B. Field. . Proceedings of the Institute of Radio Engineers, 46:240, 1958.

[14] G. B. Field. The Spin Temperature of Intergalactic Neutral Hydrogen. ApJ, 129:536-+, May 1959.

[15] S. R. Furlanetto. The global 21-centimeter background from high redshifts. MNRAS, 371:867-878, Sept. 2006. 
How the first generations of luminous baryons established the X-ray and UV backgrounds Andrei Mesinger

[16] S. R. Furlanetto et al. Astrophysics from the Highly-Redshifted $21 \mathrm{~cm}$ Line. In astro2010: The Astronomy and Astrophysics Decadal Survey, volume 2010 of ArXiv Astrophysics e-prints, pages 83-+, 2009.

[17] S. R. Furlanetto et al. Cosmology from the Highly-Redshifted $21 \mathrm{~cm}$ Line. In astro2010: The Astronomy and Astrophysics Decadal Survey, volume 2010 of ArXiv Astrophysics e-prints, pages 82-+, 2009.

[18] S. R. Furlanetto and A. Mesinger. The ionizing background at the end of reionization. MNRAS, 394:1667-1673, Apr. 2009.

[19] S. R. Furlanetto and S. P. Oh. Taxing the rich: recombinations and bubble growth during reionization. MNRAS, 363:1031-1048, Nov. 2005.

[20] S. R. Furlanetto, S. P. Oh, and F. H. Briggs. Cosmology at low frequencies: The $21 \mathrm{~cm}$ transition and the high-redshift Universe. Physics Reports, 433:181-301, Oct. 2006.

[21] S. R. Furlanetto, M. Zaldarriaga, and L. Hernquist. The Growth of H II Regions During Reionization. ApJ, 613:1-15, Sept. 2004.

[22] S. R. Furlanetto, M. Zaldarriaga, and L. Hernquist. The effects of reionization on Ly $\alpha$ galaxy surveys. MNRAS, 365:1012-1020, Jan. 2006.

[23] P. M. Geil and J. S. B. Wyithe. The impact of a percolating IGM on redshifted 21-cm observations of quasar HII regions. MNRAS, 386:1683-1694, May 2008.

[24] N. Y. Gnedin. Cosmological Reionization by Stellar Sources. ApJ, 535:530-554, June 2000.

[25] Z. Haiman, T. Abel, and M. J. Rees. The Radiative Feedback of the First Cosmological Objects. ApJ, 534:11-24, May 2000.

[26] Z. Haiman and G. L. Bryan. Was Star Formation Suppressed in High-Redshift Minihalos? ApJ, 650:7-11, Oct. 2006.

[27] Z. Haiman and R. Cen. Constraining Reionization with the Evolution of the Luminosity Function of Ly $\alpha$ Emitting Galaxies. ApJ, 623:627-631, Apr. 2005.

[28] Z. Haiman, M. J. Rees, and A. Loeb. Destruction of Molecular Hydrogen during Cosmological Reionization. ApJ, 484:985-+, Aug. 1997.

[29] A. Heger, C. L. Fryer, S. E. Woosley, N. Langer, and D. H. Hartmann. How Massive Single Stars End Their Life. ApJ, 591:288-300, July 2003.

[30] I. T. Iliev, P. R. Shapiro, P. McDonald, G. Mellema, and U.-L. Pen. The effect of the intergalactic environment on the observability of Ly $\alpha$ emitters during reionization. MNRAS, 391:63-83, Nov. 2008.

[31] I. T. Iliev, P. R. Shapiro, and A. C. Raga. Minihalo photoevaporation during cosmic reionization: evaporation times and photon consumption rates. MNRAS, 361:405-414, Aug. 2005.

[32] N. Kashikawa et al. The End of the Reionization Epoch Probed by Ly $\alpha$ Emitters at $z=6.5$ in the Subaru Deep Field. ApJ, 648:7-22, Sept. 2006.

[33] K. Kohler, N. Y. Gnedin, and A. J. S. Hamilton. Large-Scale Simulations of Reionization. ApJ, 657:15-29, Mar. 2007.

[34] M. Kuhlen and P. Madau. The first miniquasar. MNRAS, 363:1069-1082, Nov. 2005. 
How the first generations of luminous baryons established the X-ray and UV backgrounds Andrei Mesinger

[35] D. Larson et al. Seven-Year Wilkinson Microwave Anisotropy Probe (WMAP) Observations: Power Spectra and WMAP-Derived Parameters. ArXiv e-prints, Jan. 2010.

[36] A. Lidz, M. McQuinn, M. Zaldarriaga, L. Hernquist, and S. Dutta. Quasar Proximity Zones and Patchy Reionization. ApJ, 670:39-59, Nov. 2007.

[37] M. E. Machacek, G. L. Bryan, and T. Abel. Simulations of Pregalactic Structure Formation with Radiative Feedback. ApJ, 548:509-521, Feb. 2001.

[38] M. E. Machacek, G. L. Bryan, and T. Abel. Effects of a soft X-ray background on structure formation at high redshift. MNRAS, 338:273-286, Jan. 2003.

[39] S. Malhotra and J. E. Rhoads. Luminosity Functions of Ly $\alpha$ Emitters at Redshifts $z=6.5$ and z=5.7: Evidence against Reionization at z=6.5. ApJ, 617:L5-L8, Dec. 2004.

[40] A. Maselli, S. Gallerani, A. Ferrara, and T. R. Choudhury. On the size of HII regions around high-redshift quasars. MNRAS, 376:L34-L38, Mar. 2007.

[41] I. D. McGreer, A. Mesinger, and X. Fan. The first (nearly) model-independent constraint on the neutral hydrogen fraction at z 5-6. 2011.

[42] M. McQuinn, L. Hernquist, M. Zaldarriaga, and S. Dutta. Studying reionization with Ly $\alpha$ emitters. MNRAS, 381:75-96, Oct. 2007.

[43] M. McQuinn, A. Lidz, O. Zahn, S. Dutta, L. Hernquist, and M. Zaldarriaga. The morphology of HII regions during reionization. MNRAS, 377:1043-1063, May 2007.

[44] M. McQuinn, A. Lidz, M. Zaldarriaga, L. Hernquist, and S. Dutta. Probing the neutral fraction of the IGM with GRBs during the epoch of reionization. MNRAS, 388:1101-1110, Aug. 2008.

[45] A. Mesinger. Was reionization complete by z 5-6? MNRAS, 407:1328-1337, Sept. 2010.

[46] A. Mesinger, G. L. Bryan, and Z. Haiman. Ultraviolet Radiative Feedback on High-Redshift Protogalaxies. ApJ, 648:835-851, Sept. 2006.

[47] A. Mesinger, G. L. Bryan, and Z. Haiman. Relic HII regions and radiative feedback at high redshifts. MNRAS, 399:1650-1662, Nov. 2009.

[48] A. Mesinger and M. Dijkstra. Ultraviolet radiative feedback during the advanced stages of reionization. MNRAS, 390:1071-1080, Nov. 2008.

[49] A. Mesinger and S. Furlanetto. Efficient Simulations of Early Structure Formation and Reionization. ApJ, 669:663-675, Nov. 2007.

[50] A. Mesinger, S. Furlanetto, and R. Cen. 21CMFAST: a fast, seminumerical simulation of the high-redshift 21-cm signal. MNRAS, pages 1753-+, Nov. 2010.

[51] A. Mesinger and S. R. Furlanetto. Ly $\alpha$ damping wing constraints on inhomogeneous reionization. MNRAS, 385:1348-1358, Apr. 2008.

[52] A. Mesinger and S. R. Furlanetto. Ly $\alpha$ emitters during the early stages of reionization. MNRAS, 386:1990-2002, June 2008.

[53] A. Mesinger and Z. Haiman. Evidence of a Cosmological Strömgren Surface and of Significant Neutral Hydrogen Surrounding the Quasar SDSS J1030+0524. ApJ, 611:L69-L72, Aug. 2004.

[54] A. Mesinger and Z. Haiman. Constraints on Reionization and Source Properties from the Absorption Spectra of z 6.2 Quasars. ApJ, 660:923-932, May 2007. 
How the first generations of luminous baryons established the X-ray and UV backgrounds Andrei Mesinger

[55] A. Mesinger, Z. Haiman, and R. Cen. Probing the Reionization History Using the Spectra of High-Redshift Sources. ApJ, 613:23-35, Sept. 2004.

[56] S. P. Oh and Z. Haiman. Second-Generation Objects in the Universe: Radiative Cooling and Collapse of Halos with Virial Temperatures above $10^{4}$ K.ApJ,569: $558--572$, Apr. 2002.

[57] S. P. Oh and Z. Haiman. Fossil H II regions: self-limiting star formation at high redshift. MNRAS, 346:456-472, Dec. 2003.

[58] B. W. O'Shea and M. L. Norman. Population III Star Formation in a $\Lambda$ CDM Universe. II. Effects of a Photodissociating Background. ApJ, 673:14-33, Jan. 2008.

[59] E. Ripamonti, M. Mapelli, and S. Zaroubi. Radiation from early black holes - I. Effects on the neutral intergalactic medium. MNRAS, 387:158-172, June 2008.

[60] R. Salvaterra, A. Ferrara, and P. Dayal. Simulating high-redshift galaxies. ArXiv e-prints, Mar. 2010.

[61] D. Schaerer. On the properties of massive Population III stars and metal-free stellar populations. A\&A, 382:28-42, Jan. 2002.

[62] D. Schaerer. The transition from Population III to normal galaxies: Lyalpha and He II emission and the ionising properties of high redshift starburst galaxies. A\&A, 397:527-538, Jan. 2003.

[63] R. Schneider and K. Omukai. Metals, dust and the cosmic microwave background: fragmentation of high-redshift star-forming clouds. MNRAS, 402:429-435, Feb. 2010.

[64] P. R. Shapiro and H. Kang. Hydrogen molecules and the radiative cooling of pregalactic shocks. ApJ, 318:32-65, July 1987.

[65] B. D. Smith, M. J. Turk, S. Sigurdsson, B. W. O'Shea, and M. L. Norman. Three Modes of Metal-Enriched Star Formation in the Early Universe. ApJ, 691:441-451, Jan. 2009.

[66] A. Songaila. The Evolution of the Intergalactic Medium Transmission to Redshift 6. AJ, 127:2598-2603, May 2004.

[67] D. P. Stark, R. S. Ellis, J. Richard, J.-P. Kneib, G. P. Smith, and M. R. Santos. A Keck Survey for Gravitationally Lensed Ly $\alpha$ Emitters in the Redshift Range 8.5z10.4: New Constraints on the Contribution of Low-Luminosity Sources to Cosmic Reionization. ApJ, 663:10-28, July 2007.

[68] J. C. Tan and C. F. McKee. Star Formation at Zero and Very Low Metallicities. In B. W. O'Shea \& A. Heger, editor, First Stars III, volume 990 of American Institute of Physics Conference Series, pages 47-62, Mar. 2008.

[69] R. M. Thomas et al. Fast large-scale reionization simulations. MNRAS, 393:32-48, Feb. 2009.

[70] L. Tornatore, A. Ferrara, and R. Schneider. Population III stars: hidden or disappeared? MNRAS, 382:945-950, Dec. 2007.

[71] T. Totani, N. Kawai, G. Kosugi, K. Aoki, T. Yamada, M. Iye, K. Ohta, and T. Hattori. Implications for Cosmic Reionization from the Optical Afterglow Spectrum of the Gamma-Ray Burst 050904 at $\mathrm{z}=$ 6.3. PASJ, 58:485-498, June 2006.

[72] H. Trac and N. Y. Gnedin. Computer Simulations of Cosmic Reionization. ArXiv e-prints, June 2009.

[73] M. J. Turk, T. Abel, and B. O'Shea. The Formation of Population III Binaries from Cosmological Initial Conditions. Science, 325:601-, July 2009.

[74] D. Whalen, B. W. O'Shea, J. Smidt, and M. L. Norman. How the First Stars Regulated Local Star Formation. I. Radiative Feedback. ApJ, 679:925-941, June 2008. 
[75] J. H. Wise and T. Abel. Suppression of $\mathrm{H}_{2}$ CoolingintheUltravioletBackground.ApJ, 671 : $1559--1567$, Dec.2007.

[76] J. H. Wise, M. J. Turk, M. L. Norman, and T. Abel. The Birth of a Galaxy: Primordial Metal Enrichment and Population II Stellar Populations. ArXiv e-prints, Nov. 2010.

[77] S. A. Wouthuysen. On the excitation mechanism of the 21-cm (radio-frequency) interstellar hydrogen emission line. $A J, 57: 31-32,1952$.

[78] J. S. B. Wyithe and A. Loeb. A large neutral fraction of cosmic hydrogen a billion years after the Big Bang. Nature, 427:815-817, Feb. 2004.

[79] J. S. B. Wyithe, H. Yan, R. A. Windhorst, and S. Mao. A distortion of very-high-redshift galaxy number counts by gravitational lensing. ArXiv e-prints, Jan. 2011.

[80] S. Wyithe and A. Loeb. Extrapolating the Evolution of Galaxy Sizes to the Epoch of Reionization. ArXiv e-prints, Nov. 2010.

[81] N. Yoshida, K. Omukai, and L. Hernquist. Protostar Formation in the Early Universe. Science, 321:669-, Aug. 2008.

[82] N. Yoshida, A. Sokasian, L. Hernquist, and V. Springel. Early Structure Formation and Reionization in a Warm Dark Matter Cosmology. ApJ, 591:L1-L4, July 2003.

[83] O. Zahn, A. Lidz, M. McQuinn, S. Dutta, L. Hernquist, M. Zaldarriaga, and S. R. Furlanetto. Simulations and Analytic Calculations of Bubble Growth during Hydrogen Reionization. ApJ, 654:12-26, Jan. 2007.

[84] O. Zahn, A. Mesinger, M. McQuinn, H. Trac, R. Cen, and L. E. Hernquist. Comparison Of Reionization Models: Radiative Transfer Simulations And Approximate, Semi-Numeric Models. ArXiv e-prints: 1003.3455, Mar. 2010. 